\section{Microbiological criteria: Principles for their establishment and application in food quality and safety}

\author{
Liliana Pérez-Lavalle, ${ }^{1,2}$ \\ Elena Carrasco, ${ }^{1}$ Antonio Valero ${ }^{1}$ \\ ${ }^{1}$ Department of Food Science and \\ Technology, International Campus of \\ Excellence in the AgriFood Sector \\ (CeiA3), University of Córdoba, Spain; \\ ${ }^{2}$ Faculty of Basic and Biomedical \\ Sciences, Universidad Simón Bolívar, \\ Barranquilla, Colombia
}

\begin{abstract}
Legislation on food safety has led towards the standardization of food productions which, together with the existing quality certifications, aim to increase the level of protection of public health. It is recognized the need for the agri-food industry to have tools to harmonize their productions and to adequately manage their quality systems in order to improve consumers' confidence. The implementation of microbiological criteria is focused on facilitating this harmonization by enabling the discrimination of defective lots and acting as control tools at industrial level. Therefore, knowledge of the principles, components and factors influencing the efficiency of microbiological criteria may be helpful to better understand the consequences of their application. In the present study the main principles, methodologies and applications of microbiological criteria in foods are addressed for their implementation as a part of the management quality systems of agrifood industries. In addition, potential limitations and impact of microbiological criteria on food safety are discussed. Finally, an assessment of the performance of microbiological criteria at EU level in berries is described for the compliance of the socalled risk-based metrics, namely Performance Objectives and Food Safety Objectives.
\end{abstract}

\section{Introduction: perspectives and implications of industrial food quality assurance}

The agri-food industry has undergone successive modifications in the production systems in recent years in order to meet consumers' expectations towards healthier, nutritious, safer and tastier foods. The changes in food production has been based on a remarkable scientific and technological progress, genetic improvement, development of novel fertilizer compounds and pesticides or use of antibiotics in foods of animal origin. Many of these changes has given rise to more sustainable production methods leading to increase energy efficiency, improved environmental conditions or reduced waste generation, among other purposes.

Modernization and automation of industrial processes has not been simultaneously implemented in the agri-food sector since there are still industries that lack of quality standards for food quality control. This is mainly attributed to the fact that several food processes are based on artisanal methods in which producers have acquired the gained experience over different generations and they may be reluctant to shift to a more standardized and mechanized production. This fact is more evident in Small and Medium Enterprises (SMEs) which can be subjected to high variability in the implementation of hygienic procedures. This fact combined with the scarcity of knowledge of the national and international trade rules are some of the existing problems that must be tackled through a more standardized approaches oriented to quality and safety improvement. Implementation of quality certifications may serve to protect production methods as well as to increase quality assurance thus providing consumers' confidence in a more globalized market. In this framework, management of food productions is a fundamental aspect consisting of a decision-making process about the quality and safety of a food product coming from a certain lot.

Previous approaches for food quality and safety assurance have been focused on a qualitative control of the final product, allowing the acceptability of a production lot based on compliance with a certain critical limit. One of the main drawbacks of this approach is the lack of traceability to find out potential contamination sources, and the lack of dynamism in the manufacturing processes and management systems.

The use of preventive approaches facilitates the implementation of control measures along the production chain. Such approaches are based on risk analysis consisting of three interconnected components: risk assessment, risk management and risk communication (Regulation (EC) No. $178 / 2002$ ). Risk analysis is defined by the scientific procedure that serves to prioritize food quality and safety management policies and to establish public health objectives. Risk assessment is the scientific component that derives from risk analysis and consists of a systematic procedure that is
Correspondence: Antonio Valero, Department of Food Science and Technology, Faculty of Veterinary, University of Córdoba, Edificio Darwin-Anexo (C1), Campus Rabanales s/n, 14014 Córdoba, Spain.

Tel.: +34.957218516.

E-mail: avalero@uco.es

Key words: Microbial contamination, riskbased metrics, sampling plans, risk management, microbiological limit, acceptance probability, berries.

Acknowledgments: The authors are grateful to the Spanish Government (Ministerio de Economía y Competitividad, research project AGL2016-78086-R), to the 'Asociación Universitaria Iberoamericana de Postgrado (AUIP)' for their financial support and for L. Pérez Lavalle $\mathrm{PhD}$ grant; and to the Agrifood International Campus of Excellence (ceiA3).

Contributions: The authors contributed equally.

Conflict of interests: The authors declare no potential conflict of interests.

Funding: Ministerio de Economía y Competitividad, research project AGL201678086-R.

Availability of data and materials: The data that support the findings of this study are available from the corresponding author upon reasonable request.

Ethics approval and consent to participate: This research was conducted in accordance with all relevant guidelines and procedures.

Consent for publication: The manuscript does not contain any individual person's data in any form.

Received for publication: 6 September 2019. Revision received: 23 December 2019.

Accepted for publication: 7 January 2020

This work is licensed under a Creative Commons Attribution-NonCommercial 4.0 International License (CC BY-NC 4.0).

(C) Copyright: the Author(s), 2020

Licensee PAGEPress, Italy

Italian Journal of Food Safety 2020; 9:8543

doi:10.4081/ijfs.2020.8543

carried out in order to know the nature, magnitude and probability of a hazard potentially present in a food. The results derived from a risk assessment constitute a valuable base for decision-making, detection of critical points in the agri-food chain, assessment of mitigation strategies and elaboration of food trade standards (FAO/WHO, 2006).

Though risk assessment provides a quantitative measurement on the impact of 
a food hazard, the agri-food industry needs tools that can perform a formal risk assessment without the acquisition of advanced scientific knowledge or extensive information collection. One of the most valuable tools are the Food Quality and Safety Management (FQSM) systems, mainly based on the ISO 22000 standard, which specifies requirements for a FQSM system where an organization in the food chain needs to demonstrate its ability to control food safety hazards in order to ensure that food is safe at the time of human consumption. The ISO 22000 International Standard meets the specific requirements to achieve food safety based on the principles of Hazard Analysis and Critical Control Points (HACCP), and suggests applying this process through the concepts of quality management, based on the international standard ISO 9001. Both standards can be certified individually, though, they can also be implemented in an integrated way through the application of ISO 22000.

FQSM systems establish policies and control measures to guarantee food safety and public health protection (Valero et al., 2017a). These systems are based on the use of Good Hygiene and Manufacturing Practices (GHP, GMP), the principles of HACCP and self-control systems (HACCP + General Hygiene Plans or Prerequisite Programs). These tools aim to process documentation and are usually accompanied by food traceability management systems, which identify critical points along the production -consumption chain and establish corrective measures (Regulation (EC) No. 852/2004).

\section{Sampling plans as food safety management tools}

Foods generally constitute complex matrices where multiple factors interact simultaneously or deferred producing continuous modifications on the product that are often difficult to predict. The integration of preventive approaches aimed at covering the "farm-to-fork" chain must inevitably include different agents, processing steps and operations, some of them not quantifiable or predictable that complicate somehow the harmonization of the procedures of a FQSM system.

When designing a FQSM system the origin and sources of contamination of a food lot must be known, as well as the type of implicated hazard and the contamination source. For this, the so-called sampling procedures are oriented towards the measurement of a series of quality parameters and the sampling of representative portions of a given lot in order to detect the presence of contaminants.

Most sampling procedures include the selection of one or several samples from a lot, inspection or analysis of the samples and classification of the lot (as "acceptable" or "not acceptable") from the result of the inspection or sample analysis (Codex Alimentarius Commission, 2004). For this, a sampling plan constitutes a standardized procedure by which a lot is inspected and classified. Basically, the sampling plan will consider the number of units selected, as well as the maximum number of units in the lot that cannot exceed a certain level or limit ( $n$ and $\mathrm{c}$, respectively). According to the definitions stated by Codex Alimentarius, sampling plans that involve changes in the sample design, collection or analysis are called sampling schemes, while the set of sampling plans and schemes are defined as sampling systems.

In turn, sampling plans are classified into acceptance or attributes sampling plans, which measure a specific quality of a food in order to accept or reject a lot (i.e. presence of Salmonella sp. in egg products), while variables sampling plans measure a certain value or concentration in a food to check whether or not it meets certain specifications (i.e. level of $\mathrm{Sn}$ present in food containers).

In practice, acceptance sampling plans are those that are generally carried out in microbiology to detect contaminated lots with a sufficient level of confidence. In this sense, it is necessary to know that a safety margin must be established so that the sampling plan to be applied is effective enough to be used as a control measure. The sampling process of a final product can be either an effective control tool, or a procedure subject to total randomness where positive results generally have a great relevance, while the negative ones do not allow a total guarantee of absence of contamination. As an example, $1 \%$ of contaminated units in a lot may represent a seemingly small percentage in relative terms, but when it is extrapolated to a larger lot size (i.e. 100,000 units) it would mean a total of 1,000 contaminated units, thus constituting a food safety issue if that lot would be placed in the market. Therefore, the main purpose of a sampling plan is to maximize the probability of detection of contaminated lots (probability of rejection) so that it can have a high level of discrimination. In other words, it is necessary to try to minimize the percentage of units in a lot that can exceed a specified level of contamination (probability of acceptance). For its application in foods, as heterogeneous entities, the probability of detection will be strongly influenced by the sample size, the level of contamination and the variability of such contamination in the lot.

Microorganisms are defined as discrete entities, that is, as divisible entities during a finite number of times. Therefore, the extent of microbiological contamination of foods can differ depending on the type of food and the biological hazard involved. In most cases, microorganisms are heterogeneously distributed, and this makes the sampling plan a process whose nature is not intrinsically deterministic but is determined both by the predictable actions of the process and by random elements. Moreover, sampling plans are defined as stochastic processes, highly influenced by the randomness of the contamination present in the food, as well as the sampling system adopted to detect such contamination. Figure 1 exemplifies two lots with a similar level of contamination. However, the randomness of sampling itself means that in some cases no contamination

\section{Actual microbial contamination in the lot}

Figure 1. Definition of the stochastic process of sampling plans and their influence on the detection of contaminated lots. 
is detected, while in others it does, which means that the assumed contamination in the lots may be totally different from the actual contamination. To all this, analytical methods currently applied are not always able to detect $100 \%$ of the actual contamination present in the lot, especially when it is present at low levels, so one should irremediably wonder about the usefulness of sampling to ensure food safety. Efficiency of sampling plans depends mainly on the lot size, the sampling strategy, the applied analytical method and the statistical confidence to be assumed. In this sense, an effective management should imply the use of the available tools to maximize food quality and safety, and the sampling plans, as well as the microbiological criteria are just some of them.

\section{Methodologies for the derivation of microbiological criteria in foods}

In accordance with the European food legislation (Regulation (EC) No. 178/2002; Regulation (EC) No. 852/2004; Regulation (EC) No. 2073/2005), the application of microbiological criteria is oriented towards a harmonization of FQSM systems. However, for a correct interpretation of microbiological criteria it is necessary to know how they can be derived by collecting information such as: The distribution of microbial contamination in a lot; the definition of microbiological limits above which lot rejection is considered. In sampling plans by attributes of two and three classes, these values correspond to the limits $\mathrm{m}$ (lower) and M (upper); a description of the analytical method which allows to know the probability of detecting microbial contamination, as well as the limits of detection and quantification. It should be remarked that the efficiency of the sampling plans and, therefore, of the microbiological criteria is determined by the values of sensitivity (or proportion of positive samples that are actually detected by the analytical method) and specificity (or proportion of negative samples that are actually detected by the analytical method); the level of confidence to be implemented to determine the nonconformity of a lot (i.e. rejection probability $(\mathrm{Pr})=95 \%$; or acceptance probability $(\mathrm{Pa})=5 \%)$. Most of this information can be obtained from previous knowledge or historical data available, or by establishing a series of assumptions for deriving a microbiological criterion. Specifically, knowledge of the distribution of microbial contamination in a lot is often difficult to predict. However, this information is of high relevance when designing adequate sampling plans so as to optimize the probability of detection of positive samples or defective lots. Ideally, if one could spatially visualize how microorganisms are distributed into a food matrix, this would allow to have complete knowledge about the distribution of contamination in a lot (ILSI, 2010). However, this information is not often available (unless scientific studies are done at the microscopic level, which logically is not feasible). In an intermediate situation, one could know the number of cells present in a certain food location, but unfortunately, there is not much information about it either, and, in addition, the time required to

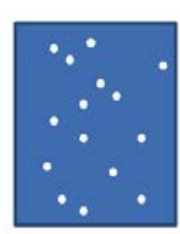

\begin{tabular}{|l|l|l|l|}
\hline 4 & 4 & 4 & 2 \\
\hline 0 & 0 & 1 & 3 \\
\hline 0 & 4 & 2 & 4 \\
\hline 2 & 1 & 1 & 0 \\
\hline
\end{tabular}
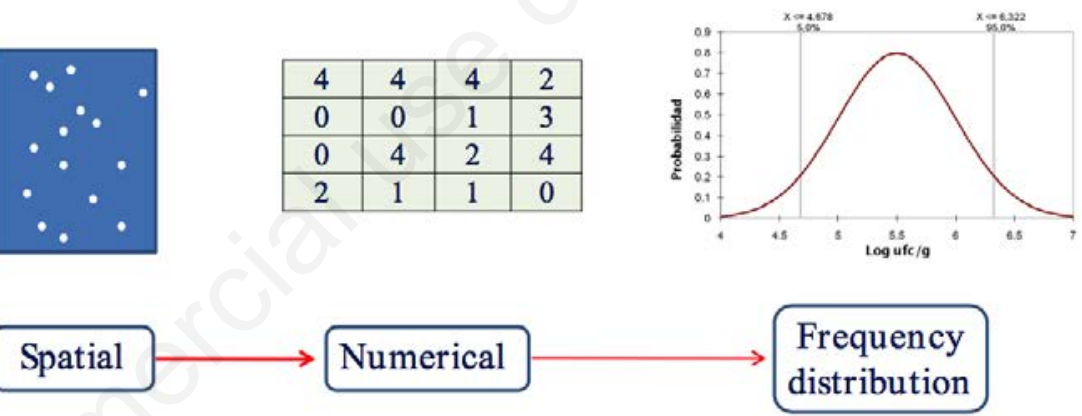

Figure 2. Representation of the microbial contamination distribution present in a food lot according to the available information (adapted from ILSI, 2010).
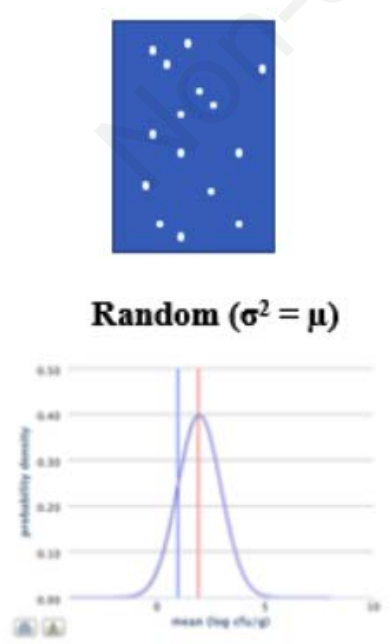

Poisson, Log normal
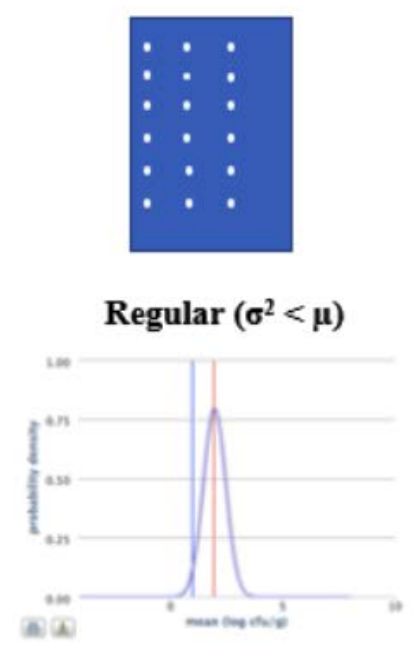

Log normal

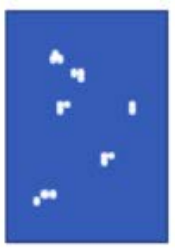

\section{Clumped $\left(\sigma^{2}>\mu\right)$}

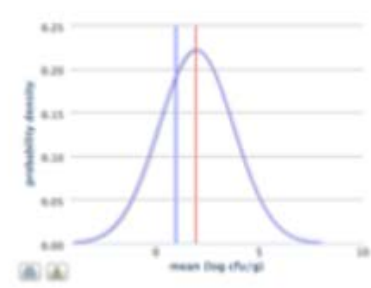

Negative Binomial, Poisson-log normal

Figure 3. Relationship between the spatial distribution of microbial contamination present in a food lot and its associated statistical distribution. 
obtain it would be unaffordable, especially when it is necessary to make decisions in a short time period. Therefore, in order to mathematically define the disposition of microbial contamination in a lot or food, one should rely on statistical distributions (Figure 2). The parameters defining each of these distributions may be used to reflect the degree of contamination of a lot, as well as the variability (dispersion) associated with the contamination in that lot. In the latter case, the distributions are used as "models" that attempt to reflect the disposition of the microorganisms in a food, that is, their level of concentration and the variability in a lot or in different lots.

The ability to model the microbial frequency distribution depends on the properties associated to each statistical distribution. Some of the most used statistical distributions for the derivation of microbiological criteria are Binomial, Normal, Poisson or Negative Binomial (Jongenburger et al., 2012). Figure 3 shows three scenarios where microbial contamination in a specific food and the statistical distribution that may be associated with such contamination are represented.

The use of the log normal distribution has been extensively described to deal with homogenous matrices and usually high concentration levels, where bacteria can be described as "continuous" entities. The parameters that can be used to define the average level of contamination of a lot and its associated variability are $\mu$ and $\sigma$, respectively. Their use is justified in cases when microbial counts are normally expressed in logarithmic scale and the standard deviation is lower than the mean. There are some published studies reflecting the importance of $\log$ normal distributions when deriving microbiological criteria (van Schothorst et al., 2009). Following the principles established by the Central Limit Theorem, any statistical distribution can approximate a normal distribution as the sample size increases, if the mean and variance ( $\mu$ and $\sigma$ ) are independent of each other. With this, the Binomial and Poisson distributions approach a normal one as the number of samples tends to infinity.

Basing on the knowledge of these properties, the efficiency of sampling plans and microbiological criteria in foods can be calculated relatively easily using the so-called Operating Characteristic Curves (Figure 4).

If microbial contamination follows a $\log$ normal distribution, in Figure 4a, the maximum allowable microbial concentration values in the lot can be estimated so that $\operatorname{Pr}=95 \%$. It can be verified that as the number of samples increases, for a value of $\mathrm{m}=1 \log \mathrm{cfu} / \mathrm{g}$, the concentration values (log $\mathrm{cfu} / \mathrm{g})$ at which $\operatorname{Pr}=95 \%$ are $1.33(\mathrm{n}=1)$; $1.15(n=2) ; 0.97(n=5) ; 0.87(n=10)$ and $0.78(n=20)$. Therefore, by increasing the number of samples from 1 to 20, the target microbial concentration at which the food lot is rejected would decrease in $0.55 \mathrm{log}$ $\mathrm{cfu} / \mathrm{g}$.

On the other hand, the influence of the standard deviation (SD) values on the efficiency of the implementation of the microbiological criteria is shown in Figure $4 b$. The standard deviation is a measure that reflects the degree of variability of the microbial contamination present in the lot. As the SD value increases, the microbiological criterion is less restrictive, having maximum allowable concentration values (log $\mathrm{cfu} / \mathrm{g})$ at which $\mathrm{Pr}=95 \%$ of $1.33(\mathrm{SD}=0.2)$; $1.66(\mathrm{SD}=0.4)$ and $2.31(\mathrm{SD}=0.8)$. This means that by increasing four times the SD values, the target concentration at which the food lot is rejected would increase in 0.98 $\log \mathrm{cfu} / \mathrm{g}$. Although the log normal approach can describe several biological phenomena, when microorganisms are randomly distributed in a food lot and contamination occurs at low levels a better approximation to the actual number of microorganisms is given using discrete distributions. A good example is the well-known singleparameter Poisson distribution which is fully defined by its location (mean; $\lambda$ ) and its dispersion (e.g. standard deviation or square root of the variance, which is equal to the mean). However, in case of having well-mixed products with low microbial concentration, or a high amount or "zero" counts, other more flexible approaches can be adopted, all derived from the Poisson distribution. For instance, the generalized Poisson distribution can be understood as a mixture of Poisson distributions providing more flexibility in order to model nonhomogeneous contamination in a food lot (Joe and Zhu, 2005). On the other hand, the "zero-inflated" Poisson distribution is used
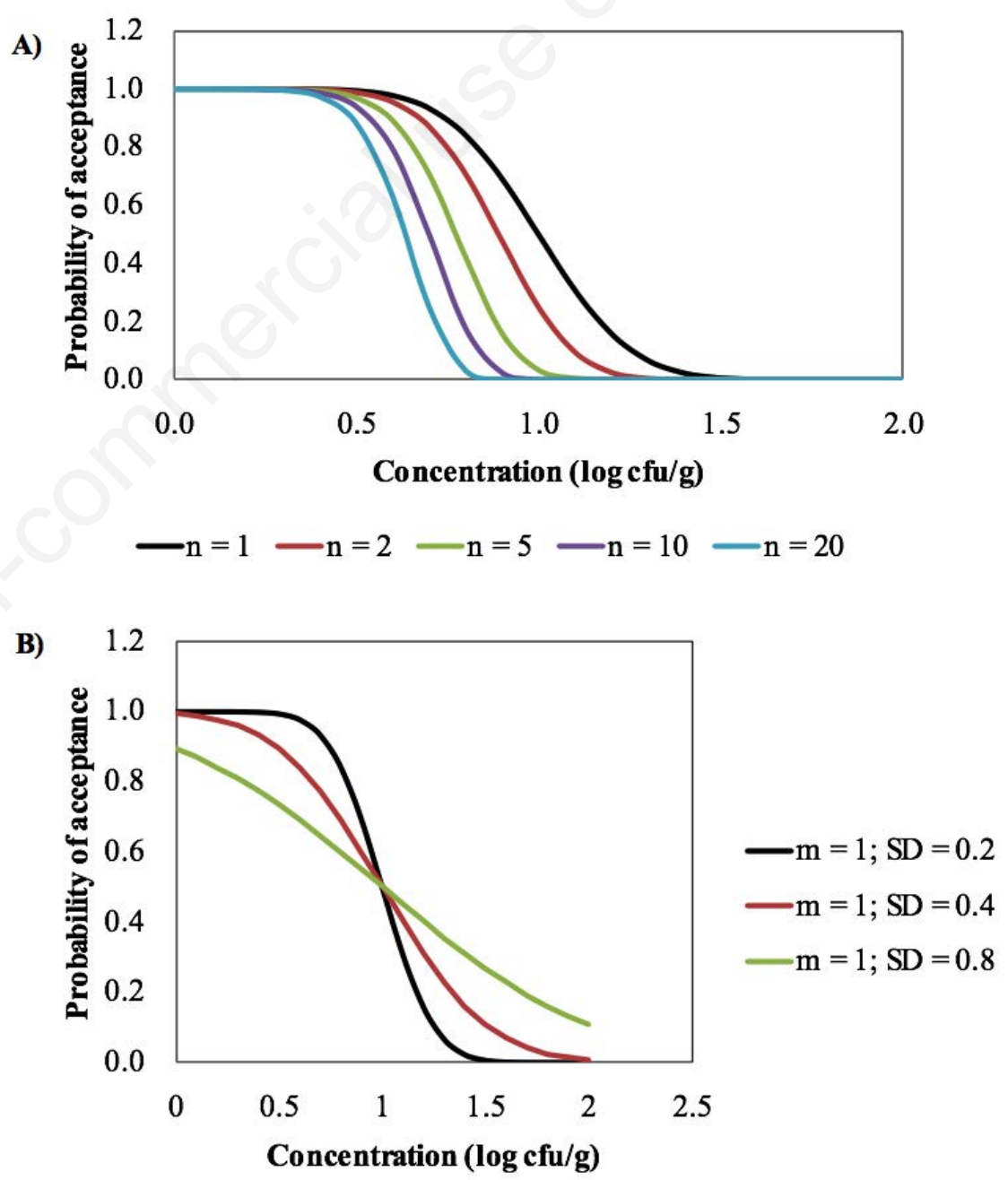

Figure 4. Operating Characteristic Curves assessing the performance of sampling plans and microbiological criteria in foods. Fig. 4a. Effect of the number of samples (n) on the probability of acceptance of the lot $(\mathrm{m}=1 \mathrm{log} \mathrm{cfu} / \mathrm{g})$; Fig. 4b. Effect of the variability of lot contamination (SD) on the probability of acceptance $(m=1 \log \mathrm{cfu} / \mathrm{g})$. 
for count data that exhibit overdispersion and excess of zeros, being successfully applied for bacterial data and stochastic risk assessment modelling (Gonzales-Barrón et al., 2010). Other discrete distributions are able to model the presence of censored data (when the observed microbial concentration is only partially known; i.e. concentration values are within a defined range but the true value is unknown), or clustering contamination, also having a good approximation to the actual proportion of non-defective units in a food lot. The Poisson-log normal distribution considers variability within lots, which is characterized by a Poisson sampling process combined with variability between lots through the assumption that concentration is log-normally distributed (Jongenburger et al., 2012). The Poissonlog normal model considers that microbial concentration in units within a lot follows a Poisson, while the true (unobserved) mean concentration varies between lots according to a $\log$ normal distribution. Finally, the assumption that the mean densities between samples can be described by a gamma distribution gives rise to the heterogeneous Poisson distribution (Poisson-gamma or negative binomial) which has been shown to be a useful distribution for a high number of zero counts or to represent cell clustering (Gonzales-Barron et al., 2014), when the standard deviation is higher than the mean (Figure 3).

In this way, by knowing the type of statistical distributions and the associated parameters, the influence of the implementation of microbiological criteria in foods can be evaluated. There are published studies where different distributions are compared to model microbial contamination in a food lot (Gonzales-Barrón and Butler, 2011; Valero et al., 2017; Reich et al., 2018). Obviously, in many cases this situation is not so trivial, so that the elaboration process, the type of biological hazard and food matrix, the step where the criterion applies etc. must be taken into account, and as abovementioned, the cost/benefit ratio resulting from the application of the microbiological criterion.

\section{The use of risk metrics for the derivation of microbiological criteria}

Microbial food safety management through risk-based approaches has led to a substantial change in the adoption of methodologies and controls by food industries along the production chain (Codex Alimentarius, 2007). These approaches have promoted a shift towards preventive strategies for food safety inspection following the recommendations made by international organizations such as EFSA, FAO/WHO or Codex Alimentarius. This requires having statistical and computer tools that attempt to predict the behaviour of microorganisms during the productionconsumption chain, as well as the need to harmonize microbiological criteria based on risk management measures.

Risk management is a process within the Risk Analysis framework that seeks to establish adequate control measures for the improvement of food quality and safety, based on the results of a risk assessment. Thus, the risk manager is a national or international government organization responsible for managing microbiological risk (EFSA, 2017).

The relationship between the requirements of food safety programs and their impact on public health is by developing targets such as Food Safety Objectives (FSO) and Performance Objectives (PO). These targets, known as risk metrics, are related to the level of risk that a society is willing to accept in order to achieve an Adequate Level of Protection or ALOP. According to ICMSF (2002), an FSO is "the maximum frequency and/or concentration of a hazard in a food at the time of consumption, which provides or contributes to reach an Appropriate Level of Protection (ALOP) for human health". A PO is instead the maximum frequency and/or concentration of a hazard in a food at a specified step in the food chain before the time of consumption that provides or contributes to an FSO or ALOP, as applicable.

The risk metrics are defined by the ICMSF (2002) and have subsequently been applied in different studies (Stringer, 2005; Valero et al., 2015; Zwietering et al., 2015) as food safety criteria for different pathogens. Risk metrics, unlike microbiological criteria, do not include information about the food, sampling plan, microbiological limit or method of analysis. They are, therefore, levels that are established based on food safety requirements from which microbiological criteria can be derived. As an example, a PO could be set to "a proportion of $99.75 \%$ of the units in a lot should not have a concentration greater than $4 \log$ $\mathrm{cfu} / \mathrm{g}$ ". If we assume that the contamination of the lot follows a normal log distribution with an average contamination value $(\mu)$ of $1.75 \mathrm{log} \mathrm{cfu} / \mathrm{g}$ and a standard deviation (SD) of $0.8 \mathrm{log} \mathrm{cfu} / \mathrm{g}$, a microbiological criterion can be derived such as $n=7 ; c=0 ; m=2 \log$ $\mathrm{cfu} / \mathrm{g}$, so that there is a probability of rejection of the lot of $95 \%$. However, on some occasions, such microbiological criteria are not feasible when implemented, so they are used primarily as tools to verify that the necessary control measures have been carried out to guarantee food safety.

\section{Practical case: evaluation of the performance of microbiological criteria for Salmonella and Escherichia coli in berries}

Berries are common edible fruits with attractive sensorial and nutritional properties since they are rich in antioxidants, phytochemicals, flavanoids, carotenoids, polyphenols, vitamins, and minerals (Giampieri et al., 2012). They are normally free from toxic chemicals and pathogenic microorganisms, primarily because of their acid $\mathrm{pH}(<4.0)$. However, these commodities are highly perishable and are consumed as ready-to-eat products. Thus, they can be subjected to microbial contamination during storage and processing leading to physico-chemical changes that may allow survival and growth or pathogenic bacteria such as Salmonella sp. or noroviruses. The potential risk posed by these pathogens have been recently studied by EFSA and translated into different opinions (EFSA, 2013; 2014) and scientific publications (Da Silva Felicio et al., 2015) since ready-to-eat fruits and vegetables were ranked as one of the most often linked to foodborne human cases of Salmonella sp. or noroviruses originated from food of nonanimal origin in the EU.

As mentioned in earlier sections, it is important to emphasize that food safety is predominantly ensured by a preventive approach (Regulation (EC) No. 852/2004). This also applies to the berries sector through the application of GAP; GMP, GHP and HACCP principles. Microbiological criteria can be either applied for validation and verification of these procedures. Regarding berries, microbiological criteria have been established for Listeria monocytogenes in all ready-to-eat foods, and for generic E. coli and Salmonella sp. in readyto-eat pre-cut fruit and vegetables and unpasteurised fruit and vegetable juices (Regulation (EC) No. 2073/2005). These criteria also apply to frozen pre-cut fruit including pre-cut berries. There are no specific microbiological criteria for fresh or frozen whole berries.

Nevertheless, there is no routine or regular monitoring of berry fruits for the presence of Salmonella sp. In the EU given the very limited prevalence data on the rates of contamination of berries by Salmonella sp. in the peer-reviewed literature only relates to fresh strawberries. There are Food Safety 
Microbiological Criteria for the absence of Salmonella $\mathrm{sp}$. in $25 \mathrm{~g}$ samples $(\mathrm{n}=5 ; \mathrm{c}=0)$ of ready-to-eat pre-cut fruit and vegetables as well as unpasteurised fruit and vegetable juices for products placed on the market during their shelf-life (Regulation (EC) No $2073 / 2005)$. For $E$. coli, it is recognized that it can serve as potential indicator of faecal contamination, thus, food contamination could lead to the presence of pathogenic bacteria on berries. At primary production level, E. coli can be found at high levels in the treated manure though it can decline in the soil when during primary processing. However, data available from published studies are not conclusive since a substantial correlation between levels of $E$. coli and Salmonella sp. presence cannot be found in berries (Delbeke et al., 2015). There are currently process hygiene microbiological criteria for $E$. coli in samples collected during the manufacturing process $(n=5 ; c=2$; $\mathrm{m}=100 \mathrm{cfu} / \mathrm{g}$ and $\mathrm{M}=1,000 \mathrm{cfu} / \mathrm{g}$ ) for readyto-eat pre-cut fruit and vegetables as well as unpasteurised fruit and vegetable juices (Regulation (EC) No 2073/2005).

The performance of microbiological criteria can be assessed when a PO or an FSO are established. Regarding Salmonella sp., presence $/ 25 \mathrm{~g}$ cannot be allowed; so that it would be equivalent to $1 \mathrm{cfu} / 25 \mathrm{~g}$. Applying a logarithmic transformation, this would lead to $-1.40 \log \mathrm{cfu} / \mathrm{g}$. For the purpose of this example, we assumed that the FSO has been set as no more than $5 \%$ of the lot units will have a pathogen concentration higher than $-1.40 \log \mathrm{cfu} / \mathrm{g}$. If we consider the overall probability of detecting a cell from any sample drawn in the lot as the product of that concentration occurs in the lot and the probability of detecting a cell (based on sample size), we are following a Poisson Log normal approach (Zwietering et al., 2015). Assuming a SD of $0.8 \mathrm{log}$ $\mathrm{cfu} / \mathrm{g}$, the mean concentration present in the lot that would fulfil with this FSO is equal to $-3.27 \log \mathrm{cfu} / \mathrm{g}$. However, if the microbiological criterion stated in the Reg. No. $2073 / 2005$ is applied, the probability of accepting the lot would be 0.95 , so this plan would be unfeasible to be applied as a control measure. In other words, by sampling 5 units from this lot we could only detect contaminated units above the microbial limit ($1.40 \log \mathrm{cfu} / \mathrm{g}$ ) with a $5 \%$ confidence. This would lead irremediably that the lot is accepted in $95 \%$ of cases. To increase the stringency of the microbiological criterion, one could i) assume a higher mean contamination and /or SD of the lot; ii) decrease the target concentration of proportion of contaminated units in the lot to achieve a higher probability of rejection. This cannot be feasible in actual terms so that the purpose of applying the criterion would be to verify that GAP, GHP/GMP or HACCP principles have been correctly carried out. For E. coli, the performance of the Process Hygiene Criterion stated in the EU Regulation can be assessed according to different levels of contamination of the food lot. Assuming that microbial contamination is log-normally distributed and the $\mathrm{SD}=0.8 \log \mathrm{cfu} / \mathrm{g}$, those lots having a mean contamination equal or higher than $2.52 \mathrm{log} \mathrm{cfu} / \mathrm{g}$ would be rejected at $95 \%$ confidence level. Taking advantage of the log normal distribution properties, one can find alternative mean and SD values that would match the required level of compliance of the microbiological criterion, as previously shown in Figure 4a-b. Although the suitability of the Process Hygiene Criterion cannot be actually assessed according to the available information, using $E$. coli as an indicator for verification of GMP and food safety management systems (including HACCP) might be useful for berries in individual processing premises where epidemiological studies indicated a higher risk of infection or at the discretion of the food business operator (EFSA, 2014).

\section{Conclusions}

In this study, the main principles for the performance of microbiological criteria and their derivation from risk management metrics have been described. Additionally, risk management decisions related to the stringency of a microbiological criterion should be made. For some specific cases, the statistical distribution of the microbial contamination is needed as well as information regarding variability within and between lots. While a risk management metric is defined as a food safety target to relate the stringency for hazard control at a specific point in the food chain to public health objectives, application of microbiological criteria allow to verify whether the food safety management system has been effectively put in place at the relevant point in the food chain. Although it is recognized that sampling schemes still offer a too high number of samples to be applied for food chain control, they constitute valid riskbased approaches for validation and verification of the safety of food lots.

\section{References}

Codex Alimentarius Commission (CAC), 2004. General Guidelines On Sampling. CAC/GL 50-2004. Accessed: 27-08-19. Available from: http://www.fao.org/
input/download/standards/10141/CXG 050s.pdf

Codex Alimentarius Commission (CAC). Working principles for risk analysis for food safety for application by governments. CAC/GL 62-2007. Accessed: 15-08-19. Available from: http://www. fao.org/input/download/standards/1075 1/CXG_062e.pdf

Commission Regulation (EC) No 178/2002 of the European Parliarment and of the Council of 28 January 2002 laying down the general principles and requirements of food law, establishing the European Food Safety Authority and laying down procedures in matters of food safety. Official Journal of the European Union No. 1, 2002.

Commission Regulation (EC) No 852/2004 of the European Parliament and of the Council of 29 April 2004 on the hygiene of foodstuffs. Official Journal of the European Union, L139 1.

Commission Regulation (EC) No. 2073/2005 of the European Parliament and of the Council of 15 November 2005 on microbiological criteria for foodstuffs. Official Journal of the European Union, L338 1-26.

Da Silva Felício MT, Hald T, Liebana E, Allende A, Hugas M, Nguyen-The C, Johannessen GS, Niskanen T, Uyttendaele M, McLauchlin J, 2015. Risk ranking of pathogens in ready-toeat unprocessed foods of non-animal origin (FoNAO) in the EU: Initial evaluation using outbreak data (20072011). Int J Food Microbiol 195:9-19.

Delbeke S, Ceuppens S, Titze Hessel C, Castro I, Jacxsens L, De Zutter L, Uyttendaele M, 2015. Microbial Safety and Sanitary Quality of Strawberry Primary Production in Belgium: Risk Factors for Salmonella and Shiga Toxin-Producing Escherichia coli Contamination. Appl Env Microbiol 81:2562-70.

EFSA BIOHAZ Panel (EFSA Panel on Biological Hazards), Ricci A, Allende A, Bolton D, Chemaly M, Davies R, Fernández Escámez PS, Girones R, Herman L, Koutsoumanis K, Lindqvist R, Robertson L, Ru G, Sanaa M, Simmons M, Skandamis P, Snary E, Speybroeck N, Ter Kuile B, Threlfall J, Wahlstrom H, Andersen JK, Uyttendaele M, Valero A, Da Silva Felício MT, Messens W, Nørrung B, 2017. Scientific Opinion on the guidance on the requirements for the development of microbiological criteria. EFSA J 15:5052.

EFSA BIOHAZ Panel (EFSA Panel on Biological Hazards), 2013. Scientific 
Opinion on the risk posed by pathogens in food of non-animal origin. Part 1 (outbreak data analysis and risk ranking of food/pathogen combinations). EFSA J 11:3025.

EFSA BIOHAZ Panel (EFSA Panel on Biological Hazards), 2014. Scientific Opinion on the risk posed by pathogens in food of non-animal origin. Part 2 (Salmonella and Norovirus in berries). EFSA J 12:3706.

Food Agriculture Organization/World Health Organization, 2006. Food Safety Risk 3949 Analysis: A Guide for National Food Safety Authorities, FAO Food and Nutrition Papers-87, 3950 FAO, Rome.

Giamperi F, Tulipani S, Álvarez-Suárez JM, Quiles JL, Mezzetti B, Battino M, 2012. The strawberry: Composition, nutritional quality, and impact on human health. Nutrition 28:9-19.

Gonzales-Barron U, Butler F, 2011. A comparison between the discrete Poissongamma and Poisson-lognormal distributions to characterise microbial counts in foods. Food Control 22:1279-86.

Gonzales-Barron U, Cadavez V, Butler F, 2014. Conducting inferential statistics for low microbial counts in foods using the Poisson-gamma regression. Food Control 37:385-94.

Gonzales-Barron U, Kerr M, Sheridan JJ, Butter F, 2010. Count data distributions and their zero-modified equivalents as a framework for modelling microbial data with a relatively high occurrence of zero counts. Int J Food Microbiol 136: 268-77.

International Commission on Microbiological Specifications for Foods (ICMSF). Microorganisms in Food 7. Microbiological testing in food safety management. 2002; NY: Kluwer Academic/Plenum.

International Life Science Institute (ILSI). Impact of Microbial Distributions on Food Safety. Commissioned by THE ILSI EUROPE Risk Analysis in Food Microbiology TASK FORCE. 2010.

Joe H, Zhu R, 2005. Generalized Poisson distribution: the property of mixture of Poisson and comparison with negative binomial distribution. Biometrical J 47:219-29.

Jongenburger I, Bassett J, Jackson T, Zwietering MH, Jewell K, 2012. Impact of microbial distributions on food safety I. Factors influencing microbial distributions and modelling aspects. Food Control 26:601-9.

Reich F, Valero A, Schill F, Bungenstock L, Klein G, 2018. Characterisation of Campylobacter contamination in broilers and assessment of microbiological criteria for the pathogen in broiler slaughterhouses. Food Control 87:60-9.

Stringer M, 2005. Summary report. Food safety objectives - role in microbiological food safety management. Food
Control 16 775-94.

Valero A, 2015. Predictive tools and strategies for establishing risk-based microbiological criteria in foods. Food Safety Challenges for Mediterranean Products. Options Méditerranéennes (Series A: Mediterranean Seminars 2015, Number 111.

Valero A, Medina E, Arroyo $\square$ López FN, 2017a. Microbial hazards and their implications in the production of table olives. Food Borne Pathogens and Antibiotic Resistance. New Jersey: John Wiley \& Sons, Inc. pp. 119-38.

Valero A, Ortiz JC, Fongaro G, Hernández M, Rodríguez-Lázaro D, 2017 b. Definition of sampling procedures for collective-eating establishments based on the distribution of environmental microbiological contamination on food handlers, utensils and surfaces. Food Control 77:8-16.

Van Schothorst M, Zwietering MH, Ross T, Buchanan RL, Cole MB, International Commission on Microbiological Specifications for Foods (ICMSF), 2009. Relating microbiological criteria to food safety objectives and performance objectives. Food Control 20:967-79.

Zwietering MH, Gorris LGM, Farber JM, 2015. Operationalising a performance objective with a microbiological criterion using a risk-based approach. Food Control 58:33-42. 